\title{
miRNAs as Essential Mediators of the Actions of Retinoic Acid in Neuroblastoma Cells
}

\author{
Salvador Meseguer, Juan-Manuel Escamilla and \\ Domingo Barettino
}

Additional information is available at the end of the chapter

http://dx.doi.org/10.5772/55444

\section{Introduction}

The discovery of microRNAs (miRNAs, miRs) led to a profound change on our vision about the regulation of gene expression in eukaryotes. MicroRNAs are an emerging class of small noncoding endogenous RNAs that participate on the fine tuning of gene expression at the posttranscriptional level. First discovered at the early 90 s in the nematode C. elegans [1], microRNAs have been involved in multiple important biological processes both in animal as in plant cells. These regulatory RNAs are transcribed as primary longer transcripts, which are then processed into 19-23-nt mature miRNAs. One strand of the mature miRNA is then incorporated into the RNA-induced silencing complex (RISC) to regulate gene expression by targeting the $3^{\prime}$-untranslated region ( $3^{\prime} \mathrm{UTR}$ ) of mRNAs with consequent translational repression and/or target mRNA degradation. This mode of action demonstrates the great regulatory potential of miRNAs, since a unique mRNA can be targeted by diverse miRNAs and conversely each miRNA may have hundreds of different target mRNAs. In recent years miRNAs have been established as important regulators of tumor development, progression and metastasis, and have demonstrated to be useful for tumor diagnosis and classification. Moreover, miRNA regulation might represent a new avenue for cancer treatment in a near future.

Neuroblastoma is the most common extracranial solid tumor in childhood and the most common tumor in infants, which originates from aberrant development of primordial neural crest cells. Several lines of evidence support the idea that microRNA deregulation could contribute to neuroblastoma pathogenesis and progression [2,3], and the usefulness of miRNA profiles for neuroblastoma diagnostics, classification and prognosis has been recently reported [4]. Neuroblastoma cell lines can be induced to differentiate in vitro by several agents, including Retinoic Acid (RA) [5, 6], the biologically active form of vitamin A. RA treatments lead to 
proliferative arrest and neuronal differentiation [5, 7] and to a reduction of the biological aggressiveness of neuroblastoma cells, by reducing their migratory and invasive abilities [8-10]. As a consequence of this, RA and its derivatives have been introduced into therapeutic protocols for neuroblastoma patients [11-13].

In this article we want to review the evidences supporting the contribution of miRNA regulation to RA-induced differentiation of neuroblastoma cells. We will show that miRNA contribute to the gene-expression changes associated with neuroblastoma cell differentiation and that specific RA-induced miRNAs target the expression of relevant genes in the context of neural differentiation. In addition RA-regulated miRNAs contribute to the reduction in the biological aggressiveness elicited by RA in vitro. We put forward the idea that miRNA regulation is part of the RA signaling pathway, and that miRNAs are essential mediators of the actions of RA in neuroblastoma cells.

\section{The molecular bases of miRNA action}

\subsection{Biogenesis of miRNAs}

miRNAs use complementary base pairing and the RNA induced silencing complex (RISC) to bind and either block translation and/or promote degradation of their target mRNAs. miRNAs are 19-22 nt-long RNA molecules transcribed mainly from non-coding regions of the genome, although some are embedded within genes, primarily as part of intronic sequences [14]. In addition, clusters of miRNAs were also found in the genome [15]. miRNAs are transcribed as large hairpin-containing molecules, called pri-miRNA, that are cleaved in the nucleus by the microprocessor complex, involving Drosha and Pasha/DGCR8 proteins [16, 17]. The result of this cleavage is a shorter precursor hairpin (approx. $70 \mathrm{nt}$ ), called pre-miRNA. Pre-miRNAs are exported through RAN GTPase and exportin-5 to the cytoplasm [18] where undergo further cleavage by Dicer to yield a transient intermediate imperfect duplex of approx 19-22 bp miRNA [19]. Subsequently, the duplex unwinds and miRNA strand is loaded into RISC complex together with proteins of the Argonaute (Ago) family [20]. The miRNA strand in RISC acts as a guide strand to find the complementary site in mRNA, and thereby suppressing the translational activity of the target mRNA. The complementary strand (known as miRNA* or as passenger strand) is degraded when the duplex is unwound, although recent evidences show that in some cases miRNA* accumulated at physiological levels and support the idea of a role for miRNA* on gene regulation [21]. (see Figure 1)

\section{2. miRNA target binding}

miRNAs interact primarily with the $3^{\prime}$-untranslated (3'UTR) region of their target mRNAs, although recent evidences show that miRNAs can also associate with sites located within the coding region of target genes [22]. In fact, complex arrays of multiple binding sites for either the same or different miRNAs located both in the 3'UTR as well as in the coding region of the target genes have been reported [23]. The base pairing of miRNA and mRNA in vertebrates requires only partial homology, with a preference for contiguous pairing occurring only at the 
seed region, located at nucleotides 2-7 of the guide strand. The lack of stringency results in a many-to-many relationship between miRNAs and mRNA targets, with the consequence that a high percentage of the genome may be regulated post-transcriptionally by a comparatively small set of miRNAs. A consequence of that is also that bioinformatic prediction of miRNA target mRNAs becomes relatively inaccurate. The guide strand binds to its complementary region in the $3^{\prime} \mathrm{UTR}$ of its target mRNA through Watson-Crick base pairing of the seed residues. Several alternative seed binding arrangements have been observed that involved different number of residues and therefore could have different binding affinity [24].

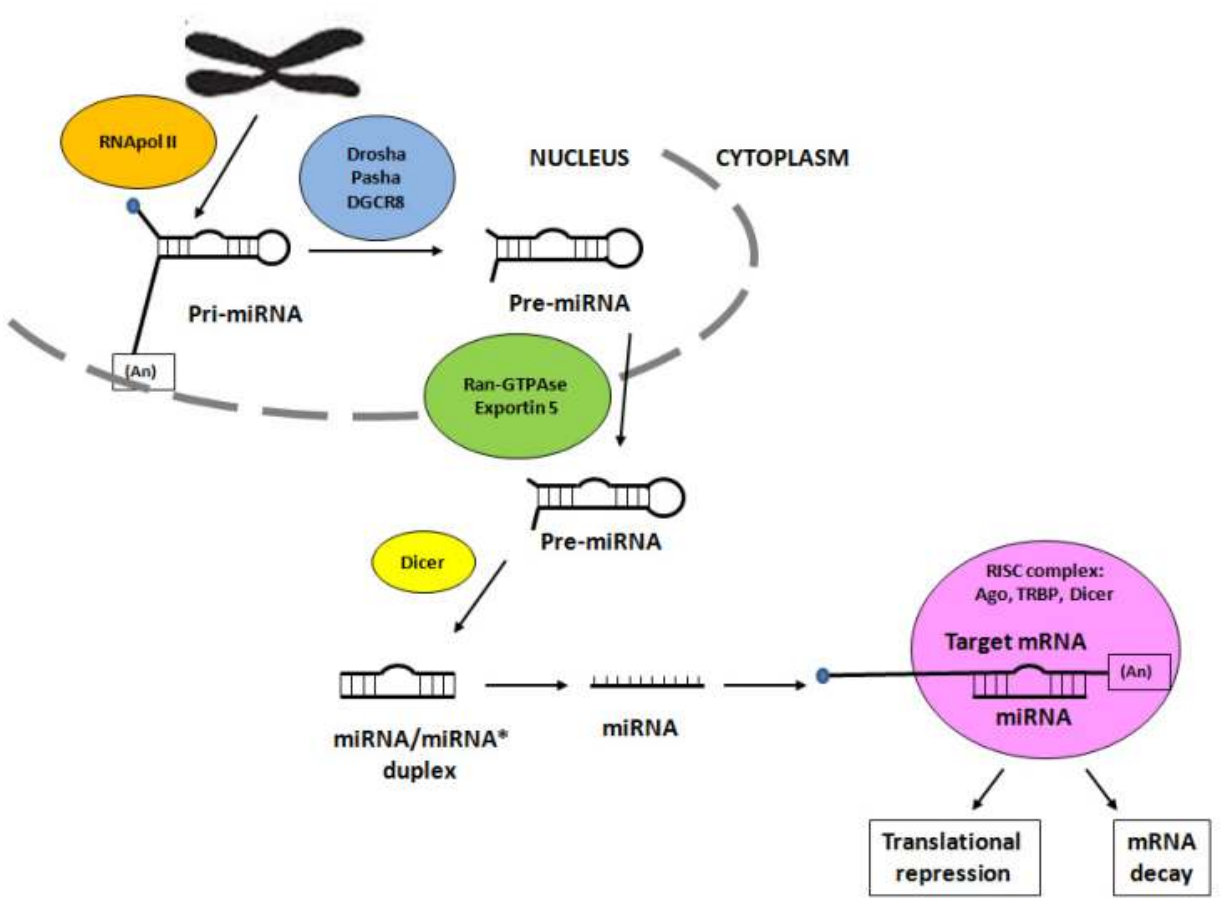

Figure 1. miRNA Biogenesis. The scheme depicts the different steps in the biogenesis of miRNAs, the enzymes involved and the intermediate miRNA forms.

\subsection{Suppression of mRNA translation and/or mRNA degradation mediated by miRNAs}

The binding of miRNA-RISC complex to its cognate mRNA target leads to mRNA silencing through suppression of mRNA translation and/or mRNA decay. [25, for review] Several mechanisms involving different protein complexes have been proposed. mRNA translation could be blocked at initiation step as well as post-initiation stages. The miRNA-RISC complex inhibits translation initiation by interfering with eIF4F-cap recognition and 40S small ribosomal subunit recruitment or by antagonizing 605 subunit joining and preventing $80 S$ ribosomal 
complex formation. The interaction of the GW182 protein with the poly(A)-binding protein (PABP) might interfere with the closed-loop formation mediated by the eIF4G-PABP interaction and thus contribute to the repression of translation initiation. The miRNA-RISC might inhibit also translation at post-initiation steps by inhibiting ribosome elongation, inducing ribosome drop-off, or promoting proteolysis of nascent polypeptides. To promote mRNA degradation, the miRNA-RISC complex interacts with the CCR4-NOT1 deadenylase complex to facilitate deadenylation of the mRNA poly(A) tail. Deadenylation requires the direct interaction of the GW182 protein with PABP. Following deadenylation, the 5 -terminal cap $\left(\mathrm{m}^{7} \mathrm{G}\right)$ is removed by the DCP1-DCP2 decapping complex. Although miRNA-mediated deadenylation followed by mRNA degradation appear to be widespread events, not all miRNA-targeted mRNAs are destabilized. miRNA-targeted translationally repressed mRNAs can accumulate in discrete cytoplasmic foci, such as P or GW bodies, or stress granules. A fraction of GW bodies co-localizes with multivesicular bodies (MVBs), membrane structures that play a role in miRNA-mediated repression. Compelling evidences support a role for miRNAs at the nucleus, acting on transcriptional regulation via chromatin remodeling and epigenetic mechanisms [26].

\section{Profiling miRNA expression during retinoic-acid-induced neuroblastoma cell differentiation}

\subsection{Profiling miRNA expression during retinoic-acid induced neuroblastoma cell differentiation}

Several studies have addressed the changes in the expression of miRNAs upon RA-dependent induction of differentiation of neuroblastoma cells, with somewhat different results depending on the cell line, treatment duration, analysis platform used, etc. [2, 27-30]. To analyze the contribution of microRNA regulation to RA-induced differentiation of neuroblastoma cells, we have studied the changes in the pattern of expression of 667 different human miRNAs upon RA treatment of SH-SY5Y neuroblastoma cells. We used miRNA profiling with TaqMan RTPCR Low Density Arrays, and we found that 452 miRNAs were expressed above detection level. From them, 42 specific miRNAs change significatively their expression levels (26 upregulated and 16 downregulated) during RA-induced differentiation (Figure 2). This suggests miRNAs as an additional post-transcriptional regulatory layer under RA control [30].

\subsection{A role for miRNAs-10a and $-10 b$ in RA-dependent regulation of neuroblastoma differentiation}

We have focused our study on the closely related miR-10a and $-10 b$, that showed the most prominent expression changes in SH-SY5Y cell line. Similar results have been reported for other neuroblastoma cell lines, like LA-N-1, LAN5 and SK-N-BE [29, 30].

Loss of function experiments with anti-sense anti-miRs antagonists could show that miR-10a and $-10 b$ contribute to the regulation of RA-induced differentiation. RA-induced neurite 
outgrowth was impaired in cells with experimentally reduced levels of miR-10a or $-10 b$, and the expression of several neural differentiation markers like Tyrosine Kinase receptors NTRK2
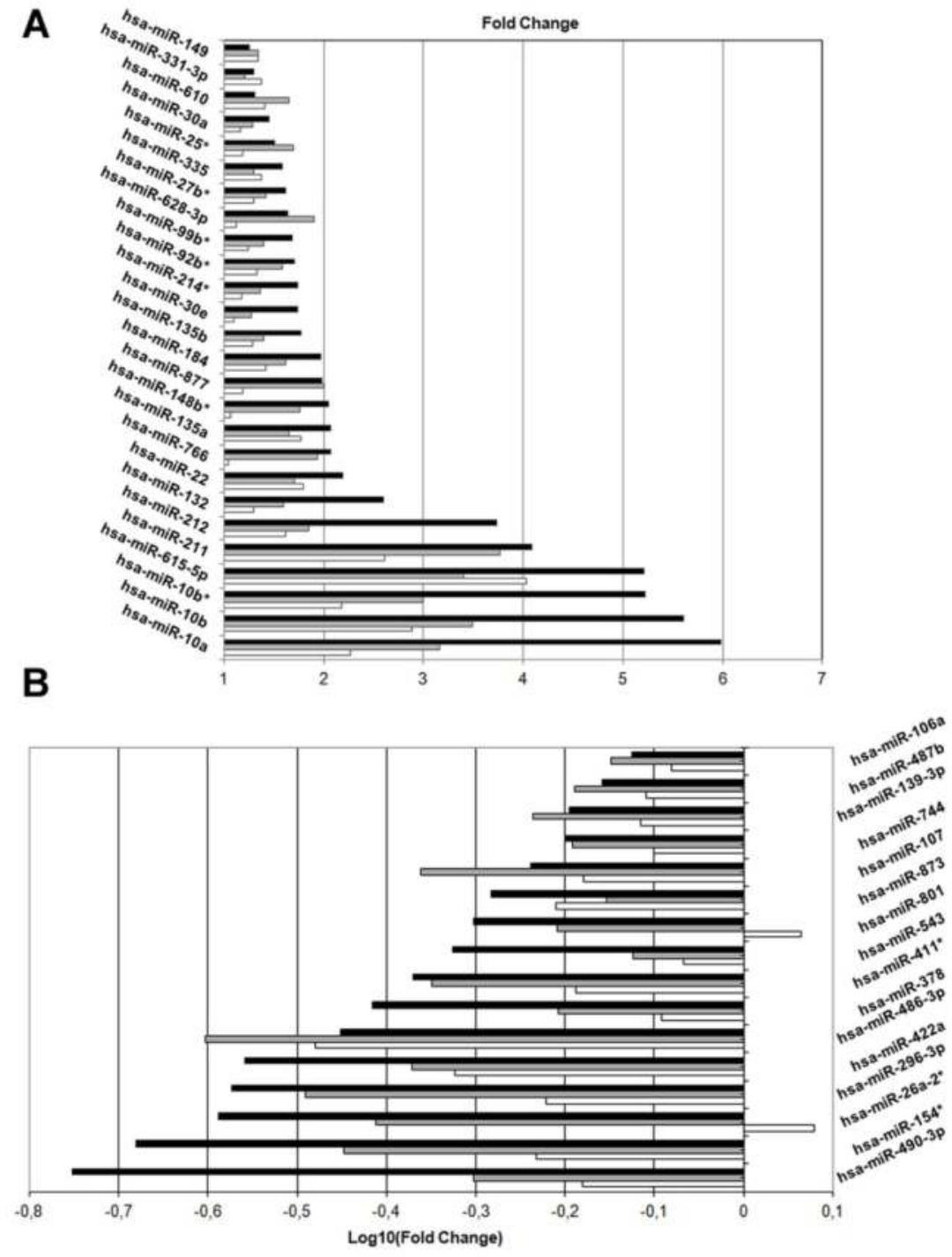

Figure 2. miRNA expression profiling in differentiating SH-SY5Y cells. Relative expression values detected in TaqMan microRNA Low Density Arrays for microRNAs with FDR $<0.05$ at least in two of the three treated versus non-treated comparisons, for upregulated (A) and downregulated miRNAs (B). The values for 24 (empty bars), 48 (grey bars) and 96 (black bars) h of RA treatment are represented. 
(trkB) and RET, GAP43, Neuron-specific Enolase (ENO2), medium-size neurofilament protein NEFM and the enzyme Tyrosine Hydroxylase (TH) was abrogated or severely impaired after suppression of miR-10a or -10b (Figure 3).

A

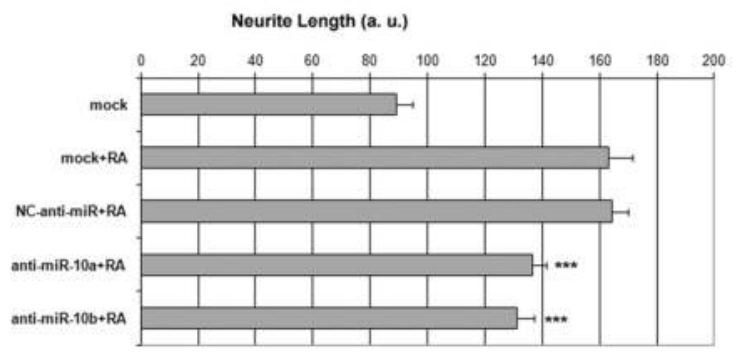

B

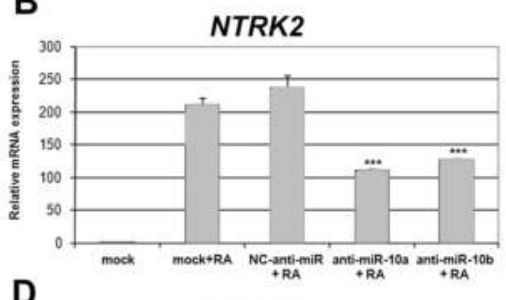

DAP43

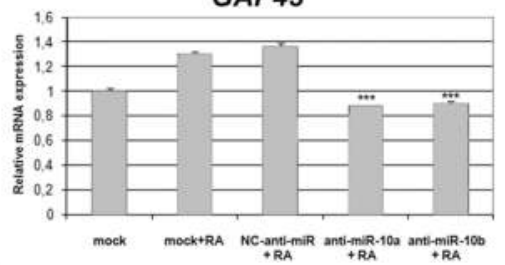

C

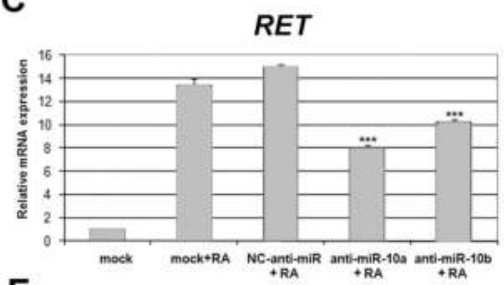

E

ENO2

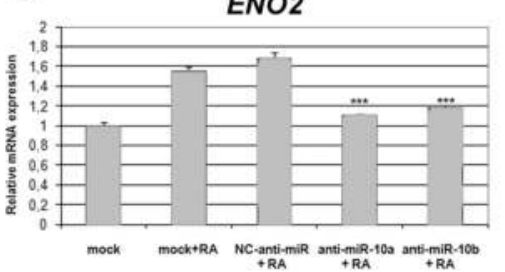

Figure 3. Knock-down of miR-10a and -10b impaired RA-induced differentiation. Blocking the action of miR-10a and $-10 \mathrm{~b}$ by transfection of their cognate anti-miRs diminished neurite outgrowth (A) and reduced the expression of neuronal differentiation markers NTRK2 (B), RET (C), GAP43 (D) and ENO2 (E), as shown by quantitative RT-PCR. (Statistical signification in the Figures: ${ }^{*}: p<0.05 ;{ }^{* *}: p<0.01 ;{ }^{* *}: p<0,001 ;$ ns: non significative)

Conversely, the downregulation of the members of the ID gene family, ID1, ID2 and ID3 was abolished in RA-treated cells transfected with anti-miR-10a and anti-miR-10b. However, miR-10a and -10b did not appear to play a relevant role in RA-induced proliferation arrest, because the strong reduction of the incorporation of ${ }^{3} \mathrm{H}$-Thymidine (to approximately $30 \%$ of the control values) and the decrease in the percentage of cells in S- phase (to $50 \%$ of the control) induced by RA treatment, was equivalent in neuroblastoma cells transfected with anti-miR-10a and anti-miR-10b [30]. However, a reduction in the cell growth in SK-N-BE neuroblastoma cells when transfected with pre-miR-10a and $-10 b$ has been reported [29]. Overexpression of miR-10 and $-10 b$ by transfecting synthetic precursor pre-miRs could not trigger full differen- 
tiation itself and although the mRNA levels of RET, NTRK2, GAP43 and ENO2 or the protein levels of NEFM and TH were slightly enhanced by transfection of pre-miR-10a and -10b, the attained expression levels for all the markers analyzed were far below those obtained by RA treatment. Similarly, ectopic expression of miR-10a and -10b led to certain increase in neurite outgrowth, but lower to that obtained for RA treatment [30]. Therefore, miR-10a and-10b appeared to be necessary but not sufficient for full neural differentiation, and consequently additional actions of RA must contribute to differentiation.

\section{3. miRNAs-10a and $-10 \mathrm{~b}$ contribute to the reduction on the biological aggressiveness of neuroblastoma cells induced by RA}

It has been reported that RA treatment of neuroblastoma cells results in a reduction in their biological aggressiveness, by decreasing their migratory and invasive abilities [8-10]. We wanted to analyze whether RA-induced expression of miR-10a and $-10 \mathrm{~b}$ could be related to the reduction in migratory and invasive potential of neuroblastoma cells. To test the migratory potential of SH-SY-5Y cells we used a modified, light-opaque Boyden chamber assay (Falcon HTS FluoroBlok, $8 \mu \mathrm{m}$ pore size). Cells were transfected with anti-miR-10a or $-10 \mathrm{~b}$ or the corresponding Negative Control anti-miR, treated with $1 \mu \mathrm{M}$ RA or vehicle in culture medium during $96 \mathrm{~h}$, and labeled in the plate with Calcein AM. Labeled cells were counted and added to the upper chamber of the Boyden chamber, and allow to migrate towards de lower chamber, filled with medium containing 10\% FBS as chemoattractant. The results show that indeed RAtreatment reduced the migration of neuroblastoma cells. However suppressing miR-10a or $-10 \mathrm{~b}$ expression not only abolished that reduction but increased migration over basal levels, supporting a contribution of RA-induced miR-10a and $10 \mathrm{~b}$ to the reduction of migratory activity produced by RA [30]. (Figure 4A)
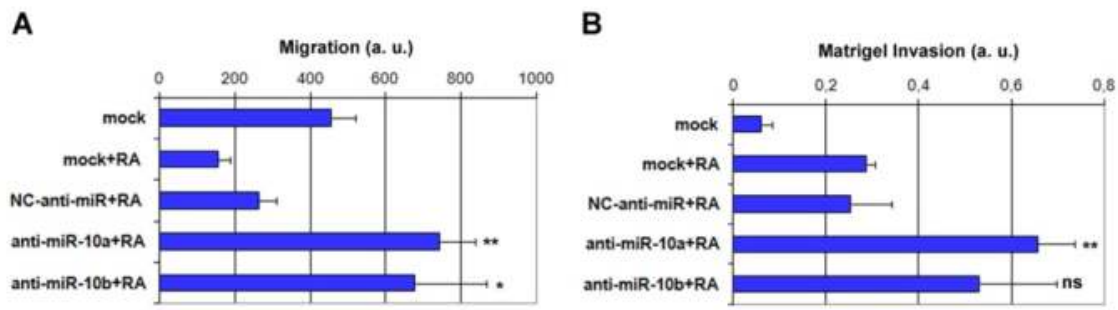

Figure 4. Involvement of miR-10a and -10b on the effects of RA in migratory and invasive potential of neuroblastoma cells. Mock-transfected cells and cells transfected with Negative Control (NC) anti-miR, anti-miR-10a or antimiR-10b were treated with $1 \mu \mathrm{M}$ RA or vehicle for $96 \mathrm{~h}$ and used in migration (A) or matrigel invasion (B) transwell assays. The graph shows a representative experiment performed in triplicate (mean \pm SD). Statistical significance was analyzed by comparing samples transfected with anti-miR-10a and -10b with those transfected with NC-anti-miR.

For invasion assays we used a similar assay, with the difference that the porous membrane separating the upper and lower chambers of the Boyden chamber was covered with BD Matrigel matrix $\left(5 \mu \mathrm{g} / \mathrm{cm}^{2}\right.$ in serum-free medium). The lower chamber contained $10 \%$ FBS as chemoattrac- 
tant to promote cell invasion. In this case RA treatment results in increased invasive potential, whereas in cells transfected with anti-miR-10a or $10 \mathrm{~b}$ the same treatment the increase in invasion induced by RA treatment is even larger, supporting the idea that the expression of miR-10a and $-10 \mathrm{~b}$ contributes to a reduction in the invasive potential [30]. (Figure 4B)

To analyze the effects of RA treatment on the metastatic potential of neuroblastoma cells we used the chicken embryo chorioallantoic membrane assays (also known as CAM assay; [31]). This assay is useful to study intravasation and metastasis in vivo, since it recapitulates all the steps of the metastatic process. In the CAM assay the cells to be tested are inoculated on the chorioallantoic membrane of 10-day-old chicken embryos. After a week, the egg is opened, the embryo is obtained and secondary organs like the lungs were dissected. The presence of human cells in the chicken organ is evaluated and quantified after obtaining their genomic DNA, by detecting the presence of human-specific Alu-sequences by Real-Time PCR (Figure 3). As this is a complex technique that requires a higher number of replicate experiments we have simplified the study to analyze only the effects of miR-10a suppression. Neuroblastoma cells could be detected in the chicken lungs after 7 days incubation. Suppression of miR-10a expression with its cognate anti-miR resulted in an increase of the metastatic cells. As expected, RA treatment led to a reduction of the number of neuroblastoma cells reaching the lungs. However this inhibitory effect of RA was abolished in cells having a reduced amount of miR-10a by transfecting the corresponding anti-miR-10a [30].

In good agreement with our results, it has been reported that miR-10a and $-10 \mathrm{~b}$ reduces the ability of neuroblastoma cells to form colonies in soft agar [29], a phenotype that is characteristic of malignant cells. All these results support the idea that miR-10a and $-10 \mathrm{~b}$ expression contribute to reduction of migratory, invasive and metastatic activities induced by RA. In a recent report it has been shown that a protein involved in cell migration, Tiam1, is targeted by miR-10b in mammary tumor cells. Overexpression of miR-10b suppresses the ability of breast carcinoma cells to migrate and invade [32]. Consistent to that, it has been reported an association between lower miR-10a expression and lower overall survival for a subclass of neuroblastoma tumors (11q- tumor cohort) [29]. However other reports seem to involve the members of the miR-10 family as promoters of migration and metastasis in different tumors [33-40]. This apparent controversy may suggest that the role of the microRNAs from the miR-10 family in tumorigenesis and metastasis would depend on their molecular targets and therefore would depend on the cellular context.

\section{Molecular targets of miR-10a and $-10 b$ in the differentiation of neuroblastoma cells}

\subsection{The search for the molecular targets for miRNAs}

The identification of molecular targets for miRNAs is a crucial step towards the understanding of miRNA function. Because an ever growing number of experimentally validated targets for miRNAs are being reported, a simple way to identify miRNA targets is to search for validated 
targets in the literature or in databases as TarBase [41]. A validated target for miR-10b in breast cancer cells is the homeobox gene HOXD10 [34, 42]. However, we could not find regulation of HOXD10 in SH-SY5Y neuroblastoma cells, when treated with RA or when the levels of miR-10a and $-10 \mathrm{~b}$ were experimentally altered [30].

A lot of effort has been made to generate computational miRNA target prediction tools [reviewed in 43], mainly based on the search for complementary sequences in the genome.

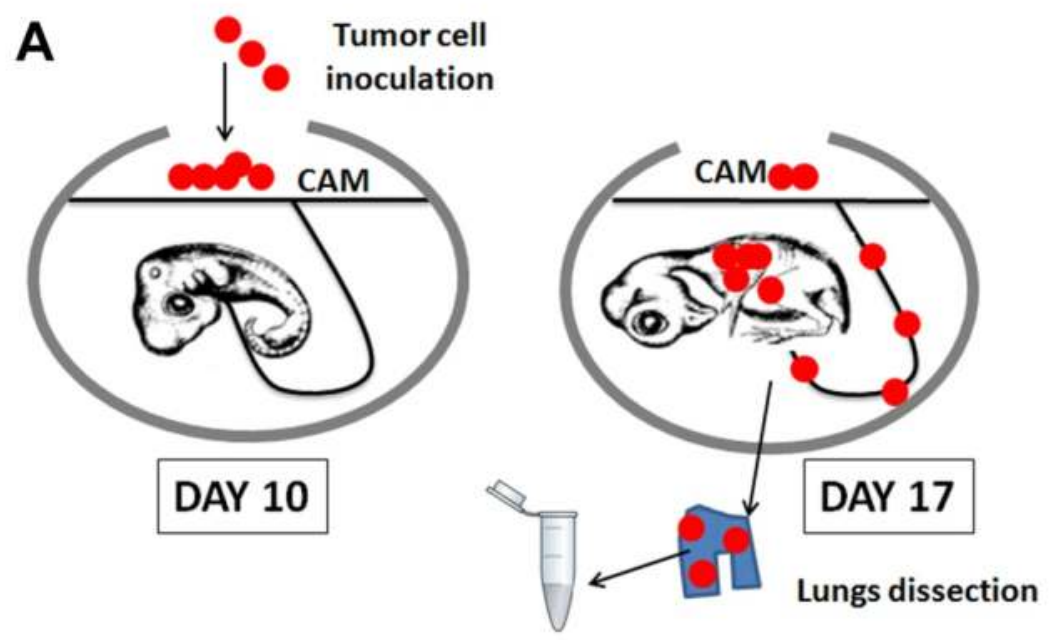

Genomic DNA extraction

B

Alu q-PCR

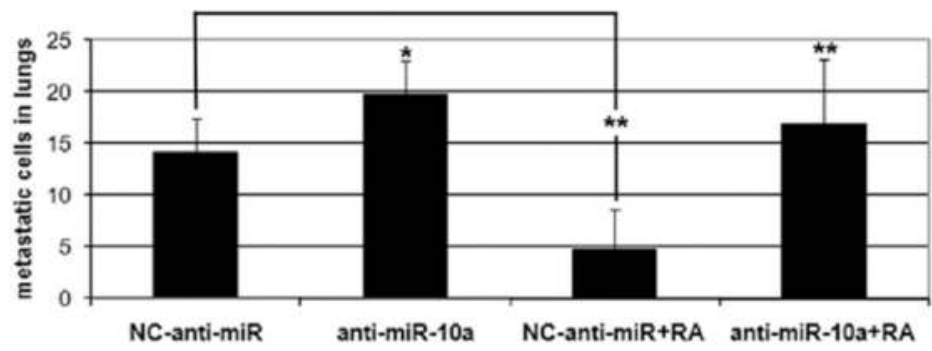

Figure 5. Chorioallantoic Membrane Metastasis Assay. (A) Schematic representation of the experiment. Cells from the different treatment groups were transferred to the upper chorioallantoic membrane of 10-day-old chicken embryos and the number of metastatized cells into the lungs evaluated 7 days later. (B) Cells transfected with Negative Control (NC) anti-miR or anti-miR-10a were treated with $1 \mu \mathrm{M}$ RA or vehicle for $96 \mathrm{~h}$ as indicated in the figure. The graph represents the values obtained from six parallel assays (mean \pm SD). Statistical significance was analyzed by comparing samples transfected with anti-miR-10a with those transfected with NC-anti-miR. In addition samples transfected with NC-anti-miR treated with vehicle were compared to those treated with RA. 
However, that is not an easy task, because short sequences are problematic for the algorithms usually developed for complementarity analysis. As indicated in 2.2, the base pairing of miRNA and mRNA in vertebrates requires only partial homology, with a preference for contiguous pairing occurring only at the "seed" region, located at nucleotides 2-7 of the guide strand, and this makes even more difficult to find the right target sequence in the genome. Several authors have approached this problem from different startpoints, using mainly complementarity analysis of the complete miRNA sequence, complementarity analysis of the seed sequence, or adding thermodynamic stability analysis of duplex sequences or 3'UTR sequence conservation to the complementarity analysis. Nowadays a set of miRNA target prediction resources are available, mainly as web-based tools. However it becomes striking to the new users of these tools how different results can be obtained when using the same sequence with different prediction tools. In addition, prediction tools generate lists of hundreds of genes for each of the miRNAs, and the fact of having sequence diversity at the 3'UTR by alternative polyadenylation sites could also complicate the analysis [for discussion, see 44].

To find relevant targets for miR-10a and-10b in neuroblastoma cells we choose to combine bioinformatic prediction tools together with experimental analysis. We created a list of potential miR-10a and $-10 \mathrm{~b}$ targets by including the common predicted genes using three different prediction resources: miRbase targets [45], TargetScan [46] and PicTar [47]. Only mRNAs that contained evolutionarily conserved miRNA binding sequences on their 3'UTR were considered. This list was crossed with the dataset of an Affymetrix microarray experiment containing the genes downregulated after $48 \mathrm{~h}$ RA treatment. In the resulting list, two members of the Arginine/serine-rich splicing factors, SFRS1 (SF2/ASF) and SFRS10 (TRA2B), as well as the nuclear receptor co-repressor NCOR2 (SMRT) were on top [30].
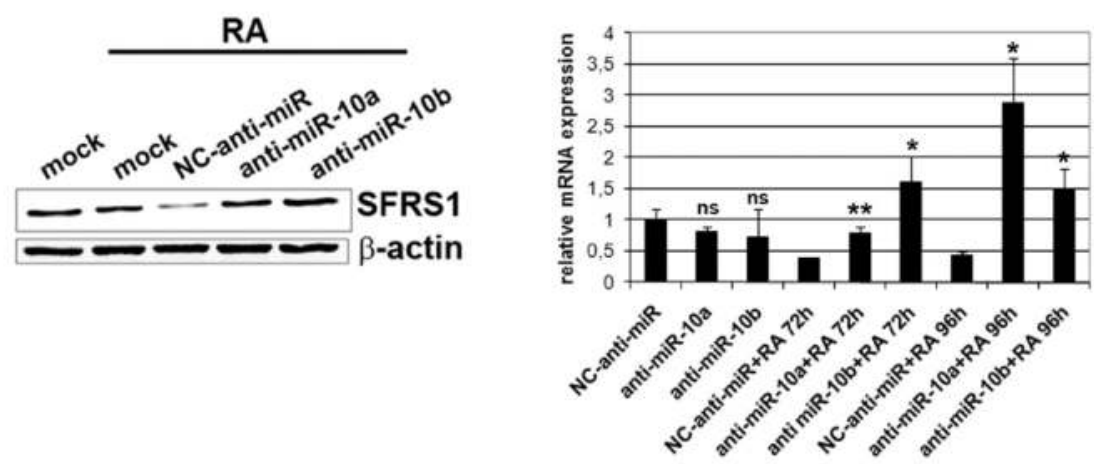

Figure 6. miR-10a/-10b knockdown leads to increased SFRS1 protein and mRNA levels in SH-SY5Y cells. (Left panel) Western blot of SFRS1 protein expression after anti-miR-10a, -10b and negative control NC-anti-miR transfection of SH-SY5Y cells followed by $1 \mu \mathrm{M}$ RA treatment. The blot was reprobed with actin beta antibodies as loading control. (Right panel). RT-qPCR analysis of SFRS1 mRNA levels in same conditions. The graph shows expression levels relative to that of RA untreated, NC-anti-miR transfected cells (mean \pm SD of a triplicate experiment). Statistical analysis for right panel was made by comparing the values from cells transfected with anti-miR-10a or -10b to those from cells transfected with NC-anti-miR; ns= non significative. 


\subsection{Regulation of SFRS1 (SF2/ASF) by miR-10a and -10b}

The regulation of SFRS1 (SF2/ASF) by miR-10a and-10b was experimentally validated at mRNA and protein levels in HeLa and SH-SY5Y cells (Figure 6). In addition regulation by miR-10a and $-10 b$ was shown in transfection experiments with reporter plasmids containing SFRS1 3'UTR sequences linked to the Luciferase gene. miR-10a and -10b are new players in the complex regulation of SFRS1 protein through a mechanism involving enhanced mRNA cleavage. In addition, we showed how changes in miR-10a and $-10 \mathrm{~b}$ expression levels may influence some molecular activities in which the product of SFRS1 is involved, such as translation enhancement of certain mRNAs and alternative splicing, that could have importance in the neural differentiation process [30] (Figure 7). We have reported that the activation of signaling pathways by RA treatment results in rapid changes in the phosphorylation pattern of SR proteins, including SFRS1 and subsequently, changes in alternative splicing selection and an increase of the translation of mRNAs containing SFRS1 binding sites take place [48]. In this context, the reduction in SFRS1 levels through miR-10a and $-10 \mathrm{~b}$ regulation could be interpreted as the closing of the feedback regulatory loop of RA on the activities of SFRS1.

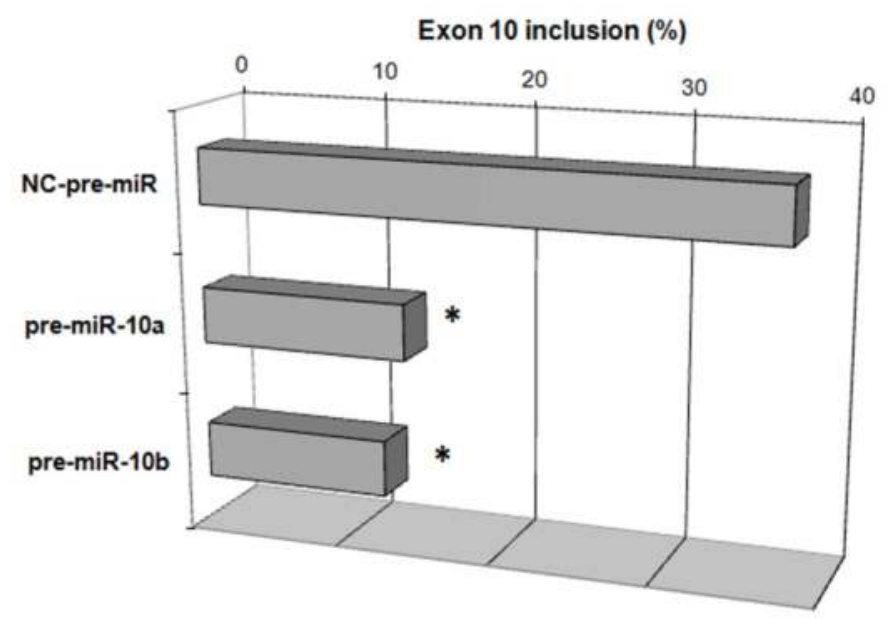

Figure 7. Experimental alteration of miR-10a and -10b levels resulted in an impairment of SFRS1 functions in the regulation of alternative splicing. Alternative splicing of tau protein exon 10 is altered by transfection of anti-miR-10a and -10b. RT-PCR was performed on RNA extracted from anti-miR-10a, -10b or negative control (NC) anti-miR transfected SH-SY5Y cells. tau Exon 10 flanking primers were used in RT-PCR reaction according to [49]. Quantification of the percentage of exon 10 inclusion. The graph shows the average from 3 independent experiments. Statistical analysis was made by comparing the values from cells transfected with pre-miR-10a or -10b to those from cells transfected with NC-pre-miR. 


\subsection{Regulation of NCOR2 (SMRT) by miR-10a and $-10 \mathrm{~b}$}

The regulation of NCOR2 by miR-10a and-10b was experimentally validated at mRNA and protein levels in SK-N-BE neuroblastoma cells. Moreover, a luciferase reporter construct containing the NCOR2 3'UTR showed a significant decrease in luciferase activity when cotransfected with mature miR-10a, $-10 \mathrm{~b}$ or $10 \mathrm{a} / 10 \mathrm{~b}$ mimics in SK-N-BE cells. This decrease in luciferase activity was completely abolished when the putative miR-10a and $-10 \mathrm{~b}$ target site was mutated in its seed sequence. Knock-down of NCOR2 expression through transfection of siRNAs to SK-N-BE cells recapitulates most of the changes induced by RA, like neurite outgrowth, proliferative arrest, expression of neural markers, downregulation of MYCN and expression of miR-10a [29]. NCOR2 acts as co-repressor in the regulation of many genes, especially as co-regulator of nuclear receptor-regulated genes. Bound to the unliganded receptor, NCOR2 maintains the promoters of nuclear receptor-regulated genes in a repressed state, and its release from the complex with the receptor upon ligand binding allows transcriptional activation [50]. It has been reported that NCOR2 represses expression of the jumonji-domain containing gene JMJD3, a direct retinoic-acid-receptor target that functions as a histone $\mathrm{H} 3$ trimethyl K27 demethylase and which is capable of activating specific components of the neurogenic program [51]. Therefore, downregulation of NCOR2 by miR-10a and $-10 \mathrm{~b}$ would potentiate the actions of RA through RARs and RXRs and could contribute to some of the changes in gene expression associated with neural differentiation.

\section{Conclusion}

MicroRNAs are essential players in the process on neural differentiation of neuroblastoma cells, and contribute to the transduction of Retinoic Acid signaling. In addition, miRNAs have been reported to participate in the pathogenesis and progression of human neuroblastoma tumors [2,3], and miRNA profiles have been recently proven to be useful for classification and prognosis [4]. Finally miRNAs open new avenues for the treatment of neuroblastoma cells, and proof of concept experiments showing a therapeutic action of miRNA-based treatments in animal models of neuroblastoma [52-54] and other tumors [33] have been reported. Therefore, we have to expect in the next years an increased interest in the study of microRNAs among the neuroblastoma researchers community.

\section{Acknowledgements}

The research work described in this article was financed through grants of the Spanish National Plan for Research, Development and Innovation (SAF2007-60780, SAF2010-15032 and SAF2011-23869), Generalitat Valenciana (ACOMP 09/212) and Genoma España to D. Barettino. S. Meseguer was the recipient of an EACR training and travel fellowship award and a CSIC I3P predoctoral fellowship/contract. 


\section{Author details}

Salvador Meseguer, Juan-Manuel Escamilla and Domingo Barettino

Instituto de Biomedicina de Valencia, Consejo Superior de Investigaciones Científicas, Valencia, Spain

\section{References}

[1] Lee, R.C., Feinbaum, R.L.,Ambros, V. The C. elegans heterochronic gene lin-4 encodes small RNAs with antisense complementarity to lin-14. Cell 1993; 75(5): 843-54.

[2] Chen, Y.,Stallings, R.L. Differential patterns of microRNA expression in neuroblastoma are correlated with prognosis, differentiation, and apoptosis. Cancer Res 2007; 67(3): 976-83.

[3] Welch, C., Chen, Y.,Stallings, R.L. MicroRNA-34a functions as a potential tumor suppressor by inducing apoptosis in neuroblastoma cells. Oncogene 2007; 26(34): 5017-22.

[4] De Preter, K., Mestdagh, P., Vermeulen, J., Zeka, F., Naranjo, A., Bray, I., Castel, V., Chen, C., Drozynska, E., Eggert, A., Hogarty, M.D., Izycka-Swieszewska, E., London, W.B., Noguera, R., Piqueras, M., Bryan, K., Schowe, B., van Sluis, P., Molenaar, J.J., Schramm, A., Schulte, J.H., Stallings, R.L., Versteeg, R., Laureys, G., Van Roy, N., Speleman, F., Vandesompele, J. miRNA expression profiling enables risk stratification in archived and fresh neuroblastoma tumor samples. Clin Cancer Res 2011; 17(24): 7684-92.

[5] Sidell, N. Retinoic acid-induced growth inhibition and morphologic differentiation of human neuroblastoma cells in vitro. J Natl Cancer Inst 1982; 68(4): 589-596.

[6] Pahlman, S., Ruusala, A.I., Abrahamsson, L., Mattsson, M.E.,Esscher, T. Retinoic acid-induced differentiation of cultured human neuroblastoma cells: a comparison with phorbolester-induced differentiation. Cell Differ 1984; 14(2): 135-144.

[7] Thiele, C.J., Reynolds, C.P.,Israel, M.A. Decreased expression of N-myc precedes retinoic acid-induced morphological differentiation of human neuroblastoma. Nature 1985; 313(6001): 404-406.

[8] Voigt, A.,Zintl, F. Effects of retinoic acid on proliferation, apoptosis, cytotoxicity, migration, and invasion of neuroblastoma cells. Med Pediatr Oncol 2003; 40(4): 205-13.

[9] Joshi, S., Guleria, R., Pan, J., DiPette, D.,Singh, U.S. Retinoic acid receptors and tissuetransglutaminase mediate short-term effect of retinoic acid on migration and invasion of neuroblastoma SH-SY5Y cells. Oncogene 2006; 25(2): 240-7. 
[10] Escamilla, J.M., Bäuerl, C., López, C.M.R., Pekkala, S.P., Navarro, S.,Barettino, D., Retinoic-Acid-Induced Downregulation of the 67 KDa Laminin Receptor Correlates with Reduced Biological Aggressiveness of Human Neuroblastoma Cells, in Neuroblastoma-Present and Future, H. Shimada, Editor. 2012, InTech: Rijeka. p. 217-232.

[11] Matthay, K.,Reynolds, C. Is there a role for retinoids to treat minimal residual disease in neuroblastoma? Br J Cancer 2000; 83(9): 1121-1123.

[12] Matthay, K.K., Reynolds, C.P., Seeger, R.C., Shimada, H., Adkins, E.S., Haas-Kogan, D., Gerbing, R.B., London, W.B.,Villablanca, J.G. Long-term results for children with high-risk neuroblastoma treated on a randomized trial of myeloablative therapy followed by 13-cis-retinoic acid: a children's oncology group study. J Clin Oncol 2009; 27(7): 1007-13.

[13] Matthay, K.K., Villablanca, J.G., Seeger, R.C., Stram, D.O., Harris, R.E., Ramsay, N.K., Swift, P., Shimada, H., Black, C.T., Brodeur, G.M., Gerbing, R.B.,Reynolds, C.P. Treatment of high-risk neuroblastoma with intensive chemotherapy, radiotherapy, autologous bone marrow transplantation, and 13-cis- retinoic acid. Children's Cancer Group. N Engl J Med 1999; 341(16): 1165-1173.

[14] Zhu, Y., Kalbfleisch, T., Brennan, M.D.,Li, Y. A MicroRNA gene is hosted in an intron of a schizophrenia-susceptibility gene. Schizophr Res 2009; 109(1-3): 86-9.

[15] Rodriguez, A., Griffiths-Jones, S., Ashurst, J.L.,Bradley, A. Identification of mammalian microRNA host genes and transcription units. Genome Res 2004; 14(10A): 1902-10.

[16] Lee, Y., Jeon, K., Lee, J.T., Kim, S.,Kim, V.N. MicroRNA maturation: stepwise processing and subcellular localization. Embo J 2002; 21(17): 4663-70.

[17] Lee, Y., Ahn, C., Han, J., Choi, H., Kim, J., Yim, J., Lee, J., Provost, P., Radmark, O., Kim, S.,Kim, V.N. The nuclear RNase III Drosha initiates microRNA processing. Nature 2003; 425(6956): 415-9.

[18] Yi, R., Qin, Y., Macara, I.G.,Cullen, B.R. Exportin-5 mediates the nuclear export of pre-microRNAs and short hairpin RNAs. Genes Dev 2003; 17(24): 3011-6.

[19] Hutvagner, G., McLachlan, J., Pasquinelli, A.E., Balint, E., Tuschl, T.,Zamore, P.D. A cellular function for the RNA-interference enzyme Dicer in the maturation of the let-7 small temporal RNA. Science 2001; 293(5531): 834-8.

[20] Meister, G., Landthaler, M., Patkaniowska, A., Dorsett, Y., Teng, G.,Tuschl, T. Human Argonaute2 mediates RNA cleavage targeted by miRNAs and siRNAs. Mol Cell 2004; 15(2): 185-97.

[21] Mah, S.M., Buske, C., Humphries, R.K.,Kuchenbauer, F. miRNA*: a passenger stranded in RNA-induced silencing complex? Crit Rev Eukaryot Gene Expr 2010; 20(2): 141-8. 
[22] Forman, J.J.,Coller, H.A. The code within the code: microRNAs target coding regions. Cell Cycle 2010; 9(8): 1533-41.

[23] Annibali, D., Gioia, U., Savino, M., Laneve, P., Caffarelli, E.,Nasi, S. A new module in neural differentiation control: two microRNAs upregulated by retinoic acid, miR-9 and -103, target the differentiation inhibitor ID2. PLoS One 2012; 7(7): e40269.

[24] Bartel, D.P. MicroRNAs: target recognition and regulatory functions. Cell 2009; 136(2): 215-33.

[25] Fabian, M.R., Sonenberg, N.,Filipowicz, W. Regulation of mRNA translation and stability by microRNAs. Annu Rev Biochem 2010; 79: 351-79.

[26] Huang, V.,Li, L.C. miRNA goes nuclear. RNA Biol 2012; 9(3): 269-73.

[27] Beveridge, N.J., Tooney, P.A., Carroll, A.P., Tran, N.,Cairns, M.J. Down-regulation of miR-17 family expression in response to retinoic acid induced neuronal differentiation. Cell Signal 2009; 21(12): 1837-45.

[28] Chen, H., Shalom-Feuerstein, R., Riley, J., Zhang, S.D., Tucci, P., Agostini, M., Aberdam, D., Knight, R.A., Genchi, G., Nicotera, P., Melino, G.,Vasa-Nicotera, M. miR-7 and miR-214 are specifically expressed during neuroblastoma differentiation, cortical development and embryonic stem cells differentiation, and control neurite outgrowth in vitro. Biochem Biophys Res Commun 2010; 394(4): 921-7.

[29] Foley, N.H., Bray, I., Watters, K.M., Das, S., Bryan, K., Bernas, T., Prehn, J.H.,Stallings, R.L. MicroRNAs 10a and 10b are potent inducers of neuroblastoma cell differentiation through targeting of nuclear receptor corepressor 2. Cell Death Differ 2011; 18(7): 1089-98.

[30] Meseguer, S., Mudduluru, G., Escamilla, J.M., Allgayer, H.,Barettino, D. MicroRNAs-10a and -10b Contribute to Retinoic Acid-induced Differentiation of Neuroblastoma Cells and Target the Alternative Splicing Regulatory Factor SFRS1 (SF2/ ASF). J Biol Chem 2011; 286(6): 4150-64.

[31] Zijlstra, A., Mellor, R., Panzarella, G., Aimes, R.T., Hooper, J.D., Marchenko, N.D.,Quigley, J.P. A quantitative analysis of rate-limiting steps in the metastatic cascade using human-specific real-time polymerase chain reaction. Cancer Res 2002; 62(23): 7083-92.

[32] Moriarty, C.H., Pursell, B.,Mercurio, A.M. miR-10b targets Tiam1: implications for Rac activation and carcinoma migration. J Biol Chem 2010; 285(27): 20541-6.

[33] Ma, L., Reinhardt, F., Pan, E., Soutschek, J., Bhat, B., Marcusson, E.G., Teruya-Feldstein, J., Bell, G.W.,Weinberg, R.A. Therapeutic silencing of miR-10b inhibits metastasis in a mouse mammary tumor model. Nat Biotechnol 2010; 28(4): 341-7.

[34] Ma, L., Teruya-Feldstein, J.,Weinberg, R.A. Tumour invasion and metastasis initiated by microRNA-10b in breast cancer. Nature 2007; 449(7163): 682-8. 
[35] Veerla, S., Lindgren, D., Kvist, A., Frigyesi, A., Staaf, J., Persson, H., Liedberg, F., Chebil, G., Gudjonsson, S., Borg, A., Mansson, W., Rovira, C.,Hoglund, M. MiRNA expression in urothelial carcinomas: important roles of miR-10a, miR-222, miR-125b, miR-7 and miR-452 for tumor stage and metastasis, and frequent homozygous losses of miR-31. Int J Cancer 2009; 124(9): 2236-42.

[36] Weiss, F.U., Marques, I.J., Woltering, J.M., Vlecken, D.H., Aghdassi, A., Partecke, L.I., Heidecke, C.D., Lerch, M.M.,Bagowski, C.P. Retinoic acid receptor antagonists inhibit miR-10a expression and block metastatic behavior of pancreatic cancer. Gastroenterology 2009; 137(6): 2136-45 e1-7.

[37] Sasayama, T., Nishihara, M., Kondoh, T., Hosoda, K.,Kohmura, E. MicroRNA-10b is overexpressed in malignant glioma and associated with tumor invasive factors, uPAR and RhoC. Int J Cancer 2009; 125(6): 1407-13.

[38] Nakata, K., Ohuchida, K., Mizumoto, K., Kayashima, T., Ikenaga, N., Sakai, H., Lin, C., Fujita, H., Otsuka, T., Aishima, S., Nagai, E., Oda, Y.,Tanaka, M. MicroRNA-10b is overexpressed in pancreatic cancer, promotes its invasiveness, and correlates with a poor prognosis. Surgery 2011; 150(5): 916-22.

[39] Lu, Y.C., Chen, Y.J., Wang, H.M., Tsai, C.Y., Chen, W.H., Huang, Y.C., Fan, K.H., Tsai, C.N., Huang, S.F., Kang, C.J., Chang, J.T.,Cheng, A.J. Oncogenic function and early detection potential of miRNA-10b in oral cancer as identified by microRNA profiling. Cancer Prev Res (Phila) 2012; 5(4): 665-74.

[40] Liu, Z., Zhu, J., Cao, H., Ren, H.,Fang, X. miR-10b promotes cell invasion through RhoC-AKT signaling pathway by targeting HOXD10 in gastric cancer. Int J Oncol 2012; 40(5): 1553-60.

[41] Vergoulis, T., Vlachos, I.S., Alexiou, P., Georgakilas, G., Maragkakis, M., Reczko, M., Gerangelos, S., Koziris, N., Dalamagas, T.,Hatzigeorgiou, A.G. TarBase 6.0: capturing the exponential growth of miRNA targets with experimental support. Nucleic Acids Res 2012; 40(Database issue): D222-9.

[42] Ma, L.,Weinberg, R.A. Micromanagers of malignancy: role of microRNAs in regulating metastasis. Trends Genet 2008; 24(9): 448-56.

[43] Maziere, P.,Enright, A.J. Prediction of microRNA targets. Drug Discov Today 2007; 12(11-12): 452-8.

[44] Clancy, J.L., Wei, G.H., Echner, N., Humphreys, D.T., Beilharz, T.H.,Preiss, T. mRNA isoform diversity can obscure detection of miRNA-mediated control of translation. Rna 2011; 17(6): 1025-31.

[45] John, B., Enright, A.J., Aravin, A., Tuschl, T., Sander, C.,Marks, D.S. Human MicroRNA targets. PLoS Biol 2004; 2(11): e363.

[46] Lewis, B.P., Shih, I.H., Jones-Rhoades, M.W., Bartel, D.P.,Burge, C.B. Prediction of mammalian microRNA targets. Cell 2003; 115(7): 787-98. 
[47] Krek, A., Grun, D., Poy, M.N., Wolf, R., Rosenberg, L., Epstein, E.J., MacMenamin, P., da Piedade, I., Gunsalus, K.C., Stoffel, M.,Rajewsky, N. Combinatorial microRNA target predictions. Nat Genet 2005; 37(5): 495-500.

[48] Laserna, E.J., Valero, M.L., Sanz, L., Sánchez del Pino, M.M., Calvete, J.J.,Barettino, D. Proteomic analysis of phosphorylated nuclear proteins underscores novel roles for rapid actions of retinoic acid in the regulation of mRNA splicing and translation. Mol Endocrinol 2009; 23(11): 1799-814.

[49] Kondo, S., Yamamoto, N., Murakami, T., Okumura, M., Mayeda, A.,Imaizumi, K. Tra2 beta, SF2/ASF and SRp30c modulate the function of an exonic splicing enhancer in exon 10 of tau pre-mRNA. Genes Cells 2004; 9(2): 121-30.

[50] Xu, L., Glass, C.K.,Rosenfeld, M.G. Coactivator and corepressor complexes in nuclear receptor function. Curr Opin Genet Dev 1999; 9(2): 140-7.

[51] Jepsen, K., Solum, D., Zhou, T., McEvilly, R.J., Kim, H.J., Glass, C.K., Hermanson, O.,Rosenfeld, M.G. SMRT-mediated repression of an H3K27 demethylase in progression from neural stem cell to neuron. Nature 2007; 450(7168): 415-9.

[52] Tivnan, A., Foley, N.H., Tracey, L., Davidoff, A.M.,Stallings, R.L. MicroRNA-184mediated inhibition of tumour growth in an orthotopic murine model of neuroblastoma. Anticancer Res 2010; 30(11): 4391-5.

[53] Tivnan, A., Tracey, L., Buckley, P.G., Alcock, L.C., Davidoff, A.M.,Stallings, R.L. MicroRNA-34a is a potent tumor suppressor molecule in vivo in neuroblastoma. BMC Cancer 2011; 11: 33.

[54] Tivnan, A., Orr, W.S., Gubala, V., Nooney, R., Williams, D.E., McDonagh, C., Prenter, S., Harvey, H., Domingo-Fernandez, R., Bray, I.M., Piskareva, O., Ng, C.Y., Lode, H.N., Davidoff, A.M.,Stallings, R.L. Inhibition of neuroblastoma tumor growth by targeted delivery of microRNA-34a using anti-disialoganglioside GD2 coated nanoparticles. PLoS One 2012; 7(5): e38129. 
